\title{
Optimization of the PLL Based TRNG Design Using the Genetic Algorithm
}

\author{
Oto Petura, Ugo Mureddu, Nathalie Bochard, Viktor Fischer \\ Univ Lyon, UJM-Saint-Etienne \\ CNRS, Laboratoire Hubert Curien UMR 5516 \\ F-42023, SAINT-ETIENNE, France
}

(oto.petura, ugo.mureddu, nathalie.bochard, fischer)@univ-st-etienne.fr

\begin{abstract}
Phase-locked loop (PLL) based true random number generator (TRNG) is very well suited for security applications using field programmable gate arrays (FPGAs) because most of FPGAs feature hardwired PLL blocks. PLL based TRNGs (PLL-TRNGs) are easy to implement and do not require manual placement or routing. The design of such TRNGs is also highly portable within the same device family. This is not the case in many other TRNG designs. However, the design of a PLLTRNGs is not a trivial task. Due to many PLL parameters, which need to be fine tuned to achieve required security and speed requirements, an exhaustive design space exploration is practically not feasible. Thus, the designers are required to go through many trial and error cycles of manual parameter tweaking and the results are still not guaranteed to be optimal. In this paper, we use a genetic algorithm (GA) based optimization to generate a suitable configuration of the PLL-TRNG, such that it is secure and reaches high output bit rate. GA optimization allows to take into account physical limits of the PLL, such as input/output frequency, and maximum voltage controlled oscillator (VCO) frequency, which avoids invalid configurations and reduces the development time. The method has proven to be very efficient and it significantly reduces the design time without compromising the security. All the presented configurations were tested on recent FPGA families and the statistical quality of the resulting TRNG configurations was verified using the AIS 31 test suite.
\end{abstract}

\section{INTRODUCTION}

True random number generators (TRNGs) constitute an essential part of cryptographic systems. The statistical quality and unpredictability of the random output, but also robustness of the generator and ability to detect attacks guarantee the security. Our goal was to design a TRNG, which can be easily implemented in field programmable gate arrays (FPGAs) and fulfill above mentioned security requirements.

We selected the phase locked loop (PLL) based TRNG (PLL-TRNG) because of its simple and comprehensive design and because of the fact that PLLs in FPGAs are physically isolated from the rest of the device, which contributes to data security. In this context, our primary goal was to select the best parameters of the generator, which will ensure the highest entropy rate at its output, and to secure the generator using an

\footnotetext{
${ }^{1}$ The article was published in the proceedings of the ISCAS 2017 conference. The published version is:

O. Petura, U. Mureddu, N. Bochard and V. Fischer, "Optimization of the PLL Based TRNG Design Using the Genetic Algorithm" 2017 IEEE International Symposium on Circuits and Systems (ISCAS), Baltimore, MD, USA, 2017, pp. 2202-2205., doi: 10.1109/ISCAS.2017.8050839
}

AIS 31 compliant approach based on the use of the stochastic model.

The PLL-TRNG was first proposed by Fischer and Drutarovsky in [1] and then enhanced by Fischer et al. in [2]. A simple stochastic model of this generator was first proposed by Simka et al. in [3]. More rigorous and more precise model was proposed later by Bernard et al. in [4].

In the recent survey published in [5], the PLL-TRNG achieved insufficient entropy rate as well as quite low output bit rate. We aim to optimize the design of the generator using a genetic algorithm (GA) in order to increase both of the above mentioned parameters as well as to reduce the design time. We then test our optimized implementation on Altera Cyclone V, Xilinx Spartan-6, and Microsemi SmartFusion ${ }^{\circledR} 2$ FPGAs.

The paper is organized as follows. In Section II, we describe basic architecture of the PLL-TRNG, its characteristics and difficulties related to its design. In Section III, we show the application of the GA on optimization of parameters of this kind of generators. In Section IV, we present the results obtained using selected GA and we discuss the obtained results in Section V. Finally, Section VI concludes the paper.

\section{THEORETICAL BACKGROUND OF THE PLL-TRNG}

The core of the PLL-TRNG is depicted in Fig. 1. The source of randomness exploited by the TRNG is the jitter of the clock signal entering the PLL $\left(c l k_{r e f}\right)$ and the tracking jitter introduced by the PLL itself. The clock signal generated by the PLL is sampled by a D flip-flop (DFF) and then $K_{D}$ subsequent samples are XOR-ed together in the decimator to make one random bit $\left(K_{M}\right.$ and $K_{D}$ are multiplication and division factors of the PLL, respectively).

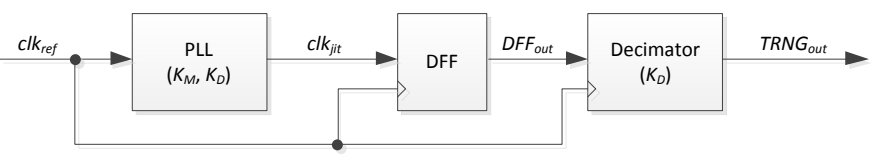

Fig. 1. General architecture of a PLL- TRNG

If the value of $K_{D}$ is odd, the output bit rate of the generator is defined as [1]:

$$
R=\frac{f_{r e f}}{K_{D}}
$$

and the sensitivity to the jitter is equal to

$$
S=\frac{K_{D}}{T_{j i t}} .
$$


TABLE I

PLl specifications for Altera Cyclone V, XilinX Spartan-6, and Microsemi SMartFusion ${ }^{\circledR} 2$ FPGA Families

\begin{tabular}{|l|c|c|c|c|c|c|l|}
\hline \multirow{2}{*}{ Parameter } & \multicolumn{2}{|c|}{ Cyclone V } & \multicolumn{2}{|c|}{ Spartan-6 } & \multicolumn{2}{c|}{ SmartFusion ${ }^{\circledR} 2$} & \multirow{2}{*}{ Description } \\
\cline { 2 - 7 } & Min & Max & Min & Max & Min & Max & \\
\hline$f_{\text {in }}[\mathrm{MHz}]$ & 5 & 500 & 19 & 540 & 1 & 200 & Input frequency of the PLL \\
\hline$M$ & 1 & 512 & 1 & 552 & 1 & 256 & Multiplication factor $(M$-counter $)$ \\
\hline$D$ & 1 & 512 & 1 & 21 & 1 & 16384 & Division factor of the input clock $(D$-counter) \\
\hline Post-VCO div. & 1 & 2 & 1 & 1 & 1 & 32 & Post-VCO division factor $(P V C O d)$ \\
\hline$C$ & 1 & 512 & 1 & 128 & 1 & 255 & Division factor of the output clock $(C$-counter $)$ \\
\hline$f_{p f d i n}[\mathrm{MHz}]$ & 5 & 325 & 19 & 500 & 1 & 200 & Input frequency of the phase frequency detector $(\mathrm{PFD)}$ \\
\hline$f_{V C O}[\mathrm{MHz}]$ & 600 & 1300 & 400 & 1000 & 500 & 1000 & Operating range of the voltage-controlled oscillator $(\mathrm{VCO})$ \\
\hline$f_{\text {out }}[\mathrm{MHz}]$ & 0 & 460 & 3.125 & 400 & 20 & 1000 & Output frequency for internal global or regional clock \\
\hline
\end{tabular}

By definition, the period relationship between the reference $\left(c l k_{r e f}\right)$ clock and the jittery clock $\left(c l k_{j i t}\right)$ is inversely proportional to the frequency relationship. Therefore, from Eq. (2) and from the PLL definition, it follows that

$$
S=f_{j i t} \cdot K_{D}=f_{\text {ref }} \cdot K_{M} .
$$

From Equations (1) and (3) it follows that to simultaneously increase sensitivity to the jitter, and thus the entropy rate, and the bit rate at the output, it is necessary to

- increase the reference frequency,

- decrease the division factor,

- increase the multiplication factor.

Unfortunately, these changes increase the internal frequencies of the PLL to the point where they might go beyond the limit of the PLL component itself. Hence, we must take into account the physical parameters of the PLL when designing the given TRNG.

As explained in [2], if the PLL-TRNG parameters are outside of intervals feasible in the given technology (i.e. given ranges of PLL parameters), two PLLs can be used. However, to reduce the total cost of the generator, our objective was to find PLL-TRNG configurations using just one PLL.

\section{A. Setup of the PLL internal structure}

Figure 2 depicts simplified general structure of PLLs available in selected FPGA families. Indeed, the PLL structure is very similar in the three FPGA device families. Concerning the architecture (but not parameters of its individual blocks), the main difference is in the presence and configuration of the post-VCO divider (VCO means the voltage controlled oscillator): while this divider is not available in the Spartan6 family, the PLLs in the Cyclone $\mathrm{V}$ family can divide the VCO output frequency by one or two, and those in the SmartFusion ${ }^{\circledR} 2$ devices can divide it by $1,2,4,816$, or 32. The number of outputs of the PLL block $(i+1)$ is also different in these devices, i.e. 4, 6, and 10 in SmartFusion ${ }^{\circledR} 2$, Spartan-6, and Cyclone V FPGA, respectively.

The physical parameters of every internal component depicted in Fig. 2 have to be taken into account in the PLLTRNG design. Table I contains the list of the parameters and their limit values documented by Altera [6], Xilinx [7], and Microsemi [8].

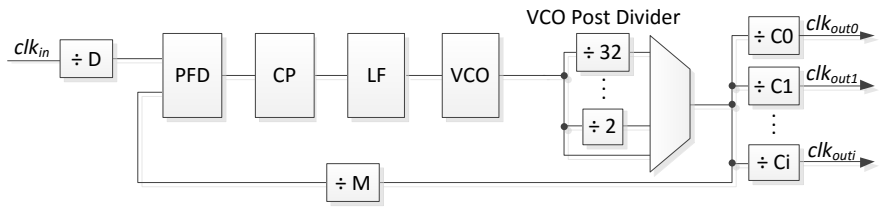

Fig. 2. Simplified general structure of the PLL block available in Altera Cyclone V, Xilinx Spartan-6, and Microsemi SmartFusion ${ }^{\circledR} 2$ FPGAs (D - input frequency division factor; $\mathrm{M}$ - multiplication factor; C - output frequency division factor; PFD - phase frequency detector; $\mathrm{CP}$ - charge pump, LF- loop filter; VCO - voltage-controlled oscillator)

The internal frequencies of the PLL are directly dependent on the chosen counter values ( $M, D$, and $C_{i}$, respectively) as well as on the input frequency of the PLL. Equations (4), (5), and (6) show the relationship between the internal frequencies and other PLL parameters:

$$
\begin{gathered}
f_{p f d i n}=\frac{f_{\text {in }}}{D}, \\
f_{V C O}=\frac{f_{\text {in }} \cdot M \cdot P V C O d}{D}, \\
f_{\text {out }}=\frac{f_{\text {in }} \cdot M}{D \cdot C} .
\end{gathered}
$$

We can conclude that the design of the PLL-TRNG depends on many parameters, which are closely related. The output bit rate of the generator (specified by Eq. (1)) and the sensitivity to the jitter (given by Eq. (3)) depend on the application needs, and the parameters described by Eq. (4) to (6) depend on selected technology and given PLL specification. Our experience shows that finding optimal PLL-TRNG parameters is not a straightforward task and some efficient strategy is therefore needed. We propose to use a genetic algorithm to solve this task.

\section{Genetic ALgorithm BASED RNG OPTIMiZation}

Genetic algorithms (GAs) belong to the group of evolutionary algorithms (EAs), which are widely used to solve searching or optimization problems. GA operates over a population of individuals. Each individual represents a solution to a problem expressed as a vector of values.

The GA starts by selecting, usually randomly, an initial set of individuals called a generation. Then it computes a fitness function which represents a "desirability" of a particular individual. The algorithm then chooses the best individuals and by the means of recombination and mutation, it generates a 
TABLE II

PARAMETERS OF THE IMPLEMENTED PLL BASED TRNGS

\begin{tabular}{|c|c|c|c|c|c|c|c|c|c|c|c|c|c|}
\hline $\begin{array}{c}\text { Config. } \\
\#\end{array}$ & $\begin{array}{c}f_{o s c} \\
{[\mathrm{MHz}]}\end{array}$ & $M$ & $D$ & $P V C O d$ & $C$ & $\begin{array}{l}f_{p f d i n} \\
{[\mathrm{MHz}]}\end{array}$ & $\begin{array}{l}f_{V C O} \\
{[\mathrm{MHz}]}\end{array}$ & $\begin{array}{c}f_{\text {out }} \\
{[\mathrm{MHz}]}\end{array}$ & $K_{M}$ & $K_{D}$ & $\begin{array}{c}R \\
{[\mathrm{Mbits} / \mathrm{s}]}\end{array}$ & $\begin{array}{c}S \\
{[\mathrm{ps}]}\end{array}$ & $\begin{array}{c}S^{-1} \\
{\left[\mathrm{ps}^{-1}\right]}\end{array}$ \\
\hline \multicolumn{14}{|c|}{ Altera Cyclone V } \\
\hline 1 & 330 & 34 & 33 & 2 & 1 & 10.00 & 680 & 340 & 34 & 33 & 10.00 & 0.011 & 89.13 \\
\hline 2 & 350 & 131 & 37 & 1 & 3 & 9.46 & 1239 & 413 & 131 & 111 & 3.15 & 0.045 & 21.81 \\
\hline 3 & 338 & 198 & 64 & 1 & 2 & 5.28 & 1046 & 522 & 198 & 128 & 2.64 & 0.067 & 14.94 \\
\hline \multicolumn{14}{|c|}{ Xilinx Spartan-6 } \\
\hline $\mathbf{1}$ & 430 & 47 & 21 & 1 & 5 & 20.476 & 962 & 192 & 47 & 105 & 4.095 & 0.020 & 49.48 \\
\hline 2 & 400 & 52 & 21 & 1 & 15 & 19.047 & 990 & 66 & 52 & 315 & 1.269 & 0.021 & 48.08 \\
\hline 3 & 430 & 48 & 12 & 1 & 27 & 20.476 & 982 & 36 & 48 & 567 & 0.758 & 0.021 & 48.45 \\
\hline \multicolumn{14}{|c|}{ Microsemi SmartFusion2 } \\
\hline 1 & 59 & 149 & 29 & 2 & 1 & 2.034 & 606 & 303 & 149 & 29 & 2.034 & 0.008 & 113.75 \\
\hline 2 & 200 & 216 & 127 & 2 & 1 & 1.574 & 680 & 340 & 216 & 127 & 1.574 & 0.043 & 23.15 \\
\hline 3 & 200 & 291 & 199 & 2 & 1 & 1.005 & 584 & 292 & 291 & 199 & 1.005 & 0.058 & 17.18 \\
\hline
\end{tabular}

new generation. This process repeats until a locally optimal solution (depending on the selected initial set of individuals) is found. Further explanation of different genetic operations and algorithms is provided in [9].

In our experiments, we used an open-source GA optimization utility provided by [10]. This utility is suitable for the GA optimization with up to 5 design variables and 5 constraints, which was sufficient in our case.

The design variables represent the parameters, which should be found (by the GA). In case of the PLL-TRNG, it is the

- input frequency $\left(f_{\text {in }}\right)$,

- multiplication factor $(M)$,

- division factor $(D)$,

- post-VCO divider $(P V C O d)$,

- output clock divider $(C)$.

The design parameters, which are not controlled directly, but which depend on design variables and need to have values within certain limits are called the constraints. In our case, the constraints were as follows:

- phase detector input frequency,

- VCO frequency,

- output frequency.

An important part of the GA optimization is the fitness function, because it determines which of the generated individuals are the best to propagate to next generations. In our case, the fitness function is a TRNG parameter, which we want to optimize for. And since security is the most critical property of a TRNG, we decided to optimize for the highest possible entropy rate, thus maximize the sensitivity to the jitter (S).

\section{IMPLEMENTATION RESULTS}

In our implementations, we used the PLL-TRNG architecture depicted in Figure 3.

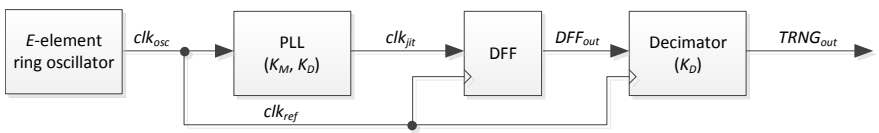

Fig. 3. Block diagram of the PLL based TRNG implemented in FPGAs
We used a ring oscillator as a reference clock source for the PLL-TRNG in order to have a configurable source of the input clock frequency.

First, we created three different GA optimization models based on Table I, one for each FPGA family. Next, we used the GA to find optimal PLL-TRNG parameters and we verified that the found parameters are indeed implementable in the corresponding family. Finally, we selected three best candidates for each of three families.

Table II presents the parameters of our optimized implementations of the PLL-TRNG ordered by the achieved bit rate. Although all the generated solutions were implementable in given families, we selected the best candidate in each family, knowing that according to our experience, to obtain high entropy rate, the sensitivity to the jitter $(S)$ should be at least 0.04 .

We selected Configuration 2 for the Altera Cyclone V family, because it had sufficient jitter sensitivity, much bigger than Configuration 1. We eliminated Configuration 3 in this family, because the division factor $\left(K_{D}\right)$ had an even value.

We selected Configuration 1 for the Xilinx Spartan-6 FPGA, because it had the highest bit rate, while the sensitivity to the jitter was very close to that of Configuration 2 and 3 (although smaller than required, i.e. smaller than 0.04).

Finally, we chose Configuration 2 for SmartFusion ${ }^{\circledR} 2$, because it was faster than Configuration 3 (while having sufficient sensitivity) and we eliminated Configuration 1 (although it was faster), because its sensitivity was not sufficient.

\section{DISCUSSION}

The use of the GA is very useful in optimization of parameters of the PLL-TRNG. This is true for two reasons:

- In order to manage the obtained result, the multiplication and division factors $K_{M}, K_{D}$ of the PLL-TRNG (and thus multiplication and division factors $D, M, C$ of the PLL) must be setup very precisely.

- Because of the fact that the technical documentation of PLL blocks, which is publicly available, is not sufficiently detailed and the user is forced to use the PLL intellectual 
property (IP) function generators, which indicate only success or failure of the PLL setup procedure and often not the direct reason of the failure.

As an example, we can mention the Microsemi FAB CCC Configurator (the Microsemi PLL IP function generator), in which the user has to enter the input and output frequencies of the PLL with the precision from two to three decimal places, in order to get required multiplication and division coefficients. This is clearly not possible using the 'failure and success' strategy. On the contrary, the application of the genetic algorithm together with the associated Excel file specified the needed coefficients and input/output frequencies and simplified largely our task.

Table II specifies sensitivities to the jitter of different PLLTRNG configurations, but the final entropy rate depends also on the clock jitter, which can vary from family to family. Therefore, we verified the quality of the generated raw random numbers using Procedure B of the AIS 31 [11] (tests T6 to T8) and we estimated the entropy rate at the generator output using the results of test T8, as it was made in [5].

Table III compares results of our implementations with those presented in [5], which used the same sources of randomness and the same FPGA families.

TABLE III

COMPARISON OF THE RESULTS OBTAINED WITH AND WITHOUT THE GA OPTIMIZATION

\begin{tabular}{|c|c|c|c|c|}
\hline Version & PLLs used & $\begin{array}{c}\text { Bit rate } \\
{[\text { Mbits/s }]}\end{array}$ & $\begin{array}{c}\text { AIS 31 } \\
(\mathrm{T} 6 \text { - T8) }\end{array}$ & $\begin{array}{c}\text { Entropy } \\
\text { estimation }\end{array}$ \\
\hline \multicolumn{5}{|c|}{ Altera Cyclone V } \\
\hline This work & 1 & 2.604 & PASS & 0.999 \\
\hline$[5]$ & 2 & 0.6 & FAIL & 0.986 \\
\hline \multicolumn{5}{|c|}{ Xilinx Spartan-6 } \\
\hline This work & 1 & 3.975 & FAIL & 0.990 \\
\hline$[5]$ & 2 & 0.44 & FAIL & 0.981 \\
\hline \multicolumn{5}{|c|}{ Microsemi SmartFusion ${ }^{\circledR} \mathbf{2}$} \\
\hline This work & 1 & 1.2 & PASS & 0.999 \\
\hline$[5]$ & 2 & 0.37 & FAIL & 0.921 \\
\hline
\end{tabular}

We can observe that the output bit streams of generators implemented in the Cyclone $\mathrm{V}$ and SmartFusion2 families passed the AIS 31 tests without any problems. It was not the case of the Spartan-6 family, because of the limitations of the PLL block. The small range of the PLL division factor made it extremely difficult to implement a TRNG using only one PLL. Since the TRNG bit rate is high enough, a possible solution would be to use an XOR based post processing. This would reduce the bit rate in favor of increasing the entropy.

The entropy estimation gave us a better knowledge about the proximity to the required statistical quality. Indeed, according to AIS 31, the Shannon entropy rate at the generator output should be higher than 0.997 . We can see that even in the case of the Spartan-6 family, our solution is much closer to the required case than that published in [5].

However, differences in bit rates are even more remarkable: comparing to [5] we increased the bit rate 3, 4, and 8 times in SmartFusion ${ }^{\circledR} 2$, Cyclone $\mathrm{V}$, and Spartan-6, respectively. Last but not least, using the GA optimization, we were able to obtain satisfying results using only one PLL. A suitable solution with only one PLL was extremely hard to find manually.

\section{CONCLUSION}

In this paper, we proposed a method of optimization of the PLL-TRNG design using a genetic algorithm. The optimized configurations reached considerably higher bit rate and their area was reduced by two by using only one PLL.

Besides increasing the bit rate and reducing the cost, the genetic algorithm was useful in increasing the entropy rate in all selected families. In two of them, Altera Cyclone V and Microsemi SmartFusion ${ }^{\circledR} 2$, the entropy rate was increased to the value, which is required by the AIS-31 standard and in the third one, Xilinx Spartan-6, the estimated entropy rate was much closer to the required value and therefore easy to increase by some low cost post-processing method.

In the near future, we plan to extend the number of input parameters and of design constrains, to manage the design of a generator using two PLLs. This could be particularly useful in the case, when a low jitter clock source must be used.

\section{ACKNOWLEDGMENT}

This work has received funding from the European Union's Horizon 2020 research and innovation programme in the framework of the project HECTOR (Hardware Enabled Crypto and Randomness) under grant agreement No 644052.

\section{REFERENCES}

[1] V. Fischer and M. Drutarovsky, "True random number generator embedded in reconfigurable hardware," in Cryptographic Hardware and Embedded Systems - CHES 2002, ser. LNCS, vol. 2523, Redwood Shores, CA, USA. Springer Verlag, 2002, pp. 415-430. [Online]. Available: http://www.springerlink.com/content/00veem7fjd2ejaqj/fulltext.pdf

[2] V. Fischer, M. Drutarovsky, M. Simka, and N. Bochard, "High performance true random number generator in Altera stratix FPLDs," in Field Programmable Logic and Application: $14^{\text {th }}$ International Conference, FPL 2004, Leuven, Belgium, August 30-September 1, 2004. Proceedings. Springer, 2004, pp. 555-564. [Online]. Available: http://www.springerlink.com/content/mx45y5j24ng3kx6q/fulltext.pdf

[3] M. Simka, M. Drutarovský, V. Fischer, and J. Fayolle, "Model of a true random number generator aimed at cryptographic applications," in International Symposium on Circuits and Systems (ISCAS 2006), 21-24 May 2006, Island of Kos, Greece, 2006.

[4] F. Bernard, V. Fischer, and B. Valtchanov, "Mathematical model of physical rngs based on coherent sampling," Tatra Mountains Mathematical Publications, vol. 45, no. 1, pp. 1-14, 2010. [Online]. Available: http://tatra.mat.savba.sk/Full/45/01be-f-v.pdf

[5] O. Petura, U. Mureddu, N. Bochard, V. Fischer, and L. Bossuet, "A survey of ais-20/31 compliant trng cores suitable for fpga devices," in 26th International Conference on Field-Programmable Logic and Applications, FPL '16, Lausanne, Switzerland, Aug. 2016.

[6] Altera, Cyclone V Device Datasheet (CV51002), 2015. [Online]. Available: www.altera.com

[7] Xilinx, Spartan-6 FPGA Clocking Resources (UG382), 2015. [Online]. Available: www.xilinx.com/support/documentation/user_guides/ug382. pdf

[8] Microsemi, SmartFusion2 and IGLOO2 Clocking Resources (UG0449), 2015. [Online]. Available: www.microsemi.com/document-portal/doc_view/ 132012-ug0449-smartfusion2-and-igloo2-clocking-resources-user-guide

[9] C. C. Coello, G. Lamont, and D. van Veldhuizen, Evolutionary Algorithms for Solving Multi-Objective Problems, 2nd ed., ser. Genetic and Evolutionary Computation. Berlin, Heidelberg: Springer, 2007. 
[10] A. Schreyer, "GA optimization for MS EXCEL," 2005. [Online]. Available: alexschreyer.net/projects/xloptim/

[11] W. Killmann and W. Schindler, "A proposal for: Functionality classes for random number generators," 2011. [Online]. Available: https://www.bsi.bund.de/SharedDocs/Downloads/DE/BSI/Zertifizierung/ Interpretationen/AIS_31_Functionality_classes_for_random_number_ generators_e.pdf?_blob=publicationFile 